\title{
The role of medium on the assembly of carbon nanotube by dielectrophoresis
}

\begin{abstract}
Dielectrophoresis (DEP) is an electrode-medium process used to control the movement of Carbon nanotubes (CNTs) within a solution under nonuniform electric fields. CNTs are typically dispersed into a solvent to form a homogeneous suspension in order to prevent their aggregation and to improve solubility. This paper starts with a short review of the techniques used to improve the compatibility of CNTs with a target medium. Then, direct and indirect variations in CNTs' velocities as a function of DEP force were investigated, under different dispersion mediums, for a rectangular electrode pair with a $10 \mu \mathrm{m}$ gap subjected to a $500 \mathrm{kHz}$ AC signal with an amplitude of $10 \mathrm{~V}$. The effects of changes in permittivity, conductivity, viscosity, and density of the medium were presented and discussed. Simulation results from this study were used to further clarify the role of the medium on the motion of CNTs during alignment using a DEP process prior to an actual alignment.
\end{abstract}

Keyword : Dielectrophoresis; Carbon nanotube; Dispersion; Assembly; Medium 